mainstream and postgraduate medical training must respond if it is to play its full part in preparing tomorrow's doctors for a more interactive future.

\section{References}

1 Academy of Medical Royal Colleges Foundation Programme Committee. The UK foundation programme curriculum. Cardiff: AoMRC, 2010.

2 UK Foundation Programme. Annual report 2009. www.foundationprogramme.nhs.uk/pages/home/key-documents\#ar
3 Academy of Medical Royal Colleges. Common competences framework for doctors. London: AoMRC, 2009.

4 Darzi A. NHS Next Stage Review: a high quality workforce. London: DH, 2008.

Address for correspondence: Professor A Maryon-Davis, Department of Primary Care and Public Health Sciences, Kings College London School of Medicine, 7th floor, Capital House, 42 Weston Street, London SE1 3QD.

Email: alan.maryon-davis@kcl.ac.uk

\title{
Improving perioperative diabetes care
}

\author{
Daniel Flanagan
}

Diabetes is the most common metabolic disorder and has a significant impact on the cost of running the NHS. With an aging population and a rising prevalence of obesity the problem will continue to grow. ${ }^{1}$ Specialist diabetes services have traditionally been based in secondary care often with diabetes centres situated within hospitals but, despite this, little attention has specifically been given to care of people with diabetes in hospital beds.

Although diabetes physicians will often participate in the general medical admissions rota and will perform an important role in looking after general medical inpatients, they may have little involvement in the care of diabetes for the vast majority of people with this condition admitted to hospital. This is despite the knowledge that diabetes control is difficult in the face of intercurrent illness. A number of studies have shown that diabetes leads to increased in-hospital morbidity and increased length of stay. ${ }^{2,3}$ There is little published evidence that improving diabetes care improves outcomes but what evidence there is supports this. ${ }^{4,5}$ The recent National Diabetes Inpatient Audit showed that between $15 \%$ and $20 \%$ of hospital beds are occupied by people with diabetes. ${ }^{6}$ There is increasing awareness that clinical care needs to focus on this large group of patients. $^{7}$

Over recent years a number of groups have shown that providing specialist diabetes services in hospital can significantly improve diabetes care. ${ }^{8}$ Work carried out in the Plymouth

Daniel Flanagan, consultant physician, Plymouth Diabetes Service, Derriford Hospital, Plymouth, Devon
Diabetes Service has shown that a specialist diabetes team can improve quality of care. A dedicated team of specialist nurses and doctors supported by dietetics can significantly improve inpatient diabetes care. Short-term quality of care is difficult to measure in hospital - a crude measure is the length of hospital stay. Having established an inpatient diabetes team in the service in late 2002, a significant reduction in length of stay between 2002 and 2006 was recorded. The mean reduction in length of stay was 0.7 days for a hospital diabetes population of approximately 6,000 in Derriford Hospital. This equates to approximately 4,000 less bed days per year occupied by people with diabetes. In addition to the clinical benefit to individuals this represents a major efficiency saving for the hospital, something that is being strongly encouraged in the current financial climate.

Disappointingly this work did not show a reduction in the length of stay for people attending hospital for elective procedures. As this group represents about $50 \%$ of people in hospital the service was very keen to understand the reasons why. For patients attending for planned procedures the path of the admission is often established before the person comes into hospital. Decisions are made about whether or not the person can be treated as a day case or whether admission to hospital overnight is required. It was clear from the service's work on the wards that for a proportion of patients with diabetes, admission to hospital had probably not been necessary but intervention when in hospital was too late. A project to map the pathway taken by people with diabetes attending for elective procedures was established to improve this process. Four key stages at which diabetes specialist input can improve care were identified. 


\section{Pre-assessment}

One of the key problems was that patients were unnecessarily admitted to hospital for a procedure because they had a diagnosis of diabetes. The British Association of Day Surgery guidelines define which surgical procedures should be performed as day cases. It is clear that a high proportion of patients that were being admitted could be treated and discharged on the same day if suitable plans were developed for managing their condition. Early identification of diabetes is important, ideally at the time of referral from primary care, to allow adequate time to optimise diabetes care and develop an individual treatment strategy.

\section{The day of admission}

A close working relationship between diabetes specialists and anaesthetists is essential to optimising perioperative diabetes care. It is important that hospitals develop institutional plans for managing patients on the variety of locally used insulin regimens. Patients need to be clear about what they are expected to do before attending hospital and what will happen to them once they arrive. Diabetes expertise needs to be available to help when problems arise. If this expertise is not available, a common response is to commence an intravenous insulin infusion. This almost inevitably results in an unexpected overnight stay in hospital.

\section{Postoperative ward care}

Postoperative diabetes care is often neglected. This is a time when glucose control is likely to be difficult due to metabolic changes associated with surgery and poor appetite. Experience would suggest that treatment of both hyper- and hypoglycaemia is often delayed. A proactive approach to identifying patients that may experience difficulties may significantly improve diabetes care.

\section{Follow-up of diabetes care to allow early discharge}

Problems with glycaemic control often result in prolonged admission to hospital. If diabetes expertise can be provided out of hospital this may allow the patient to go home with close support available to ensure that glucose control is safely managed. The key to this is to combine both the inpatient and outpatient diabetes specialist roles.

The Plymouth diabetes team focused on the above four key areas using the successful strategies that had been previously used within the inpatient diabetes service. In addition to providing a troubleshooting role and acting as diabetes champions the importance of staff education, protocol development and audit of the work were emphasised.

The day case rates and length of stay of people with diabetes over the 12-month period of the pilot were compared with the previous 12 months. One of the main goals of the project was to try to improve day case surgery rates for people with diabetes. A $35 \%$ increase in this measure was seen for people with diabetes. Over the same period there was a more modest rise of $14 \%$ for the general population. In addition a reduction in mean length of stay from 2.64 days to 2.30 days for people with diabetes admitted electively to hospital was recorded.

Although this pilot project did require the employment of additional specialist staff, the underlying principles of diabetes specialists planning diabetes care in close cooperation with anaesthetic and surgical staff can be applied with minimal additional resource. Jointly developing local protocols and guidelines for the management of the various aspects of elective diabetes care has in itself highlighted a number of areas where care could be improved. This is probably the most important first step to take.

\section{References}

1 Centers for Disease Control and Prevention. National diabetes fact sheet: general information and facts about diabetes in the United States, 2007. Atlanta: US Department of Health, 2007.

2 McAlister FA, Majumdar SR, Blitz S et al. The relation between hyperglycemia and outcomes in 2,471 patients admitted to the hospital with community-acquired pneumonia. Diabetes Care 2005;28:810-5.

3 Schiele F, Scotes-Genon V, Seronde MF et al. Predictive value of admission hyperglycaemia on mortality in patients with acute myocardial infarction. Diabet Med 2006;23:1370-6.

4 Van den Berghe G, Wouters P, Weekers F et al. Intensive insulin therapy in the critically ill patients. N Engl J Med 2001;345:1359-67.

5 Gandhi GY, Nuttall GA, Abel MD et al. Intraoperative hyperglycemia and perioperative outcomes in cardiac surgery patients. Mayo Clin Proc 2005;80:862-6.

6 Rayman G, Taylor CG, Malik R et al. Initial feedback from clinical teams participating in the National Diabetes Audit Day. Diabet Med 2010;27(Suppl 1):P375.

7 Think Glucose. Inpatient care for people with diabetes. www.institute.nhs.uk/quality_and_value/think_glucose

8 Flanagan D, Moore E, Baker S, Wright D, Lynch P. Diabetes care in hospital - the impact of a dedicated inpatient care team. Diabet Med 2008;25:147-51.

Address for correspondence: Dr D Flanagan, Department of Endocrinology, Level 9, Derriford Hospital, Plymouth PL6 8DH. Email: daniel.flanagan@phnt.swest.nhs.uk 